\title{
THE STEFAN PROBLEM WITH HEATING: APPEARANCE AND DISAPPEARANCE OF A MUSHY REGION
} BY

\author{
M. BERTSCH, P. DE MOTTONI AND L. A. PELETIER
}

\begin{abstract}
We consider a material which is initially in the solid state and then, due to heating, starts to melt. We describe the appearance of a so-called mushy region, i.e., a region in which the material is in neither a solid nor a liquid state. The main result is that after a finite time the mushy region has disappeared and only the solid and the liquid phases have remained.
\end{abstract}

1. Introduction. In this paper we study the problem

$$
\begin{cases}u_{t}=(\phi(u))_{x x}+f(u) & \text { in }(0,1) \times \mathbf{R}^{+}, \\ \phi(u)(0, t)=(\phi(u))_{x}(1, t)=0 & \text { in } \mathbf{R}^{+}, \\ u(x, 0)=u_{0}(x) & \text { in }[0,1],\end{cases}
$$

in which the functions $\phi, f$, and $u_{0}$ satisfy

H1. $\phi$ is locally Lipschitz continuous and nondecreasing on $\mathbf{R}, \phi(0)=0$ and for some $0<\alpha<\beta<\infty$,

$$
\begin{aligned}
& \phi \in C^{1}(\mathbf{R} \backslash(\alpha, \beta)) \quad \text { and } \quad \phi^{\prime}>0 \quad \text { on } \mathbf{R} \backslash(\alpha, \beta) \text {, } \\
& \phi=\phi(\alpha)=\phi(\beta) \text { on }[\alpha, \beta] \text {. }
\end{aligned}
$$

For convenience we choose $\phi(\alpha)=1$.

H2. $f$ is locally Lipschitz continuous and positive on $\mathbf{R}^{+}$.

H3. $u_{0} \in L^{\infty}(0,1),\left\|u_{0}\right\|_{\infty}<\alpha$ and $u_{0}$ is nondecreasing.

H4. $\phi, f$ and $u_{0}$ are such that the associated stationary problem

$$
\left\{\begin{array}{l}
(\phi(v))^{\prime \prime}+f(v)=0 \text { on }(0,1) \\
\phi(v)(0)=(\phi(v))^{\prime}(1)=0
\end{array}\right.
$$

has a solution $w$, which is minimal in the set $\left\{w \geqslant u_{0}\right\}$, and $\phi(w)(1)>1$. In addition, $u_{0}$ is a subsolution of Problem II.

Hypotheses $\mathrm{H} 1-\mathrm{H} 4$ will be assumed throughout the paper; we shall refer to them collectively as hypothesis $\mathrm{H}$.

More general versions of Problem I, all having in common that $\phi^{\prime}$ vanishes on an interval, have been considered in a previous paper [3]. Existence, uniqueness and regularity properties of solutions were established there, and many references were given.

Received by the editors March 7, 1985.

1980 Mathematics Subject Classification. Primary 35K65, 35R35.

(C)1986 American Mathematical Society $0002-9947 / 86 \$ 1.00+\$ .25$ per page 
The connection of Problem I with the Stefan problem was also discussed in [3]. Then the function $u$ represents the enthalpy, $\phi(u)$ the temperature and $\phi(u)=1$ is the melting temperature of the material. If $\phi(u)<1$, the material is in the solid phase; and if $\phi(u)>1$, it is in the liquid phase. The two phases are separated by a set $\mathscr{M}$ in which $\phi(u)=1$. The interior $\mathscr{M}$ of $\mathscr{M}$, if it is not empty, is usually referred to as the mushy region.

Let

$$
\mathscr{M}(t)=\{x \in[0,1]:(x, t) \in \mathscr{M}\}
$$

and let $\stackrel{\mathscr{M}}{ }(t)$ be defined similarly.

In the absence of interior heating $(f=0)$ it has been known for some time $[4,7]$ that

$$
\stackrel{\mathscr{M}}{ }(0)=\varnothing \Rightarrow \stackrel{\mathscr{M}}{ }(t)=\varnothing \quad \forall t \geqslant 0 .
$$

In $[3,6]$ it was shown that if the boundary condition at $x=1$ is replaced by $\phi(u)(1, t)=A>1$, then, for any-quite general-initial enthalpy profile $u_{0}$, there exists a time $T_{0}$ such that

$$
\stackrel{\mathscr{M}}{ }(t)=\varnothing \quad \forall t \geqslant T_{0} .
$$

Thus, in this case, mushy regions disappear in finite time.

If there is interior heating ( $f>0$ ), the situation is quite different. If an initially solid material is heated, and the heating is so strong that it results in melting of parts of the material, then, as was shown in [3], a mushy region emerges; i.e., there exists a time $T_{1}>0$ such that

$$
\stackrel{\mathscr{M}}{ }(t)=\varnothing \quad \text { for } 0<t \leqslant T_{1}
$$

and

$$
\stackrel{\mathscr{M}}{(t)} \neq \varnothing \text { for } T_{1}<t<T_{1}+\tau,
$$

and a lower bound for $\tau \in \mathbf{R}^{+}$can be given.

Numerical work of Atthey [2] and an example studied analytically by Ughi [8] suggest that the set $\mathscr{\mathscr { M }}$ does not extend indefinitely into the future, but that there exists a time $T^{*}$ such that

$$
\stackrel{\mathscr{M}}{ }(t)=\varnothing \quad \forall t \geqslant T^{*}
$$

i.e., the mushy region disappears again in finite time. A conjecture to this effect was already made in [3].

The object of this paper is to prove this conjecture for solutions of Problem I with functions $\phi, f$ and $u_{0}$ satisfying hypothesis $\mathrm{H}$. The source function $f$ and the initial profile $u_{0}$ are so chosen that $u(x, t)$ is increasing in $t$ and in $x$, and that at $x=1$ the medium passes in time from solid to mush and then to liquid. Thus, interfaces appear at $x=1$ - first between solid and mush, and then between mush and liquid - which recede monotonically towards $x=0$. This leads to the situation sketched in Figure 1. 


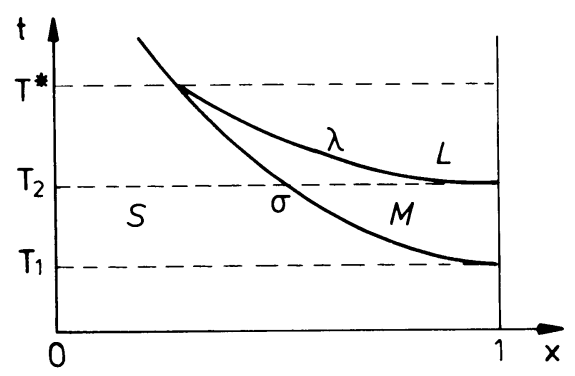

FIGURE 1. Disappearance of $\mathscr{M}$

While proving the conjecture we also prove that these solid/mush and mush/liquid interfaces are continuous, and that the function $u(\cdot, t)$ is continuous across the solid-mush interface (but not across the mush-liquid interface). The fact that this interface is receding plays a crucial role here. In [3] examples were given which show that $u(\cdot, t)$ need not be continuous when the solid/mush interface advances.

In the following we shall borrow some of the terminology of the literature on the Stefan problem and call

$$
\mathscr{S}:=\left\{(x, t) \in[0,1] \times \overline{\mathbf{R}}^{+}: \phi(u)(x, t)<1\right\}
$$

the solid region,

$$
\mathscr{L}:=\left\{(x, t) \in[0,1] \times \overline{\mathbf{R}}^{+}: \phi(u)(x, t)>1\right\}
$$

the liquid region, and the interior $\mathscr{M}$ of the set

$$
\mathscr{M}:=\left\{(x, t) \in[0,1] \times \mathbf{R}^{+}: \phi(u)(x, t)=1\right\}
$$

the mushy region.

In view of the degeneracy of the equation in Problem I, we need to define solutions in a weak sense. For convenience we write

$$
\Omega=(0,1), \quad Q_{T}=\Omega \times(0, T], \quad Q=\Omega \times \mathbf{R}^{+} .
$$

Definition 1.1. A (weak) solution $u(t)$ of Problem I on $[0, T]$ is a function $u:[0, T] \rightarrow L^{1}(\Omega)$ with the properties

$$
\begin{aligned}
& u \in C\left([0, T] ; L^{1}(\Omega)\right) \cap L^{\infty}\left(Q_{T}\right) \\
& \int_{\Omega} u(t) \psi(t)-\int_{\Omega} u_{0} \psi(0)-\int_{0}^{t} \int_{\Omega}\left(u \psi_{t}+\phi(u) \psi_{x x}+f(u) \psi\right)=0
\end{aligned}
$$

for all $t \in[0, T]$ and all $\psi \in C^{2,1}\left(\bar{Q}_{T}\right), \psi \geqslant 0, \psi=0$ at $x=0$ and $\psi_{x}=0$ at $x=1$.

$A$ solution on $[0, \infty)$ is a solution on $[0, T]$ for every $T>0$. A sub( super $)$ solution is defined by (i) and (ii) with equality replaced by $\leqslant(\geqslant)$.

$A$ (weak) solution (respectively sub- and supersolution) of the associated stationary problem is defined in an analogous way.

To study the qualitative behaviour of weak solutions, we shall frequently employ comparison arguments, using a family of sub- and supersolutions, specifically tailored to this problem. 
Because $u_{0}$ is nondecreasing, we find that at any time $t>0$, the temperature profile $\phi(u)(\cdot, t)$ is nondecreasing, and thus proceeding from $x=0$ to $x=1$, one may pass from solid to mush and from mush to liquid, but not from mush back to solid. Thus we are led to introduce the following family of classical sub- and supersolutions: given $0 \leqslant \tau_{s}<\tau_{l} \leqslant T$, we introduce two functions

$$
\sigma:\left[\tau_{s}, T\right] \rightarrow[0,1], \quad \lambda:\left[\tau_{l}, T\right] \rightarrow[0,1]
$$

with $\sigma\left(\tau_{s}\right)=\lambda\left(\tau_{l}\right)=1$ (the solid/mush and the mush/liquid interfaces), and we define the sets

$$
\begin{aligned}
\mathscr{S}_{T}:= & \left\{(x, t) \in \bar{Q}_{T}: t<\tau_{s}\right\} \cup\left\{(x, t) \in \bar{Q}_{T}: 0 \leqslant x<\sigma(t), \tau_{s} \leqslant t \leqslant T\right\}, \\
\mathscr{L}_{T}:= & \left\{(x, t) \in \bar{Q}_{T}: \lambda(t)<x \leqslant 1, \tau_{l}<t \leqslant T\right\}, \\
\mathscr{M}_{T}:= & \left\{(x, t) \in \bar{Q}_{T}: \sigma(t) \leqslant x \leqslant 1, \tau_{s} \leqslant t<\tau_{l}\right\} \\
& \cup\left\{(x, t) \in \bar{Q}_{T}: \sigma(t) \leqslant x \leqslant \lambda(t), \tau_{l} \leqslant t \leqslant T\right\} .
\end{aligned}
$$

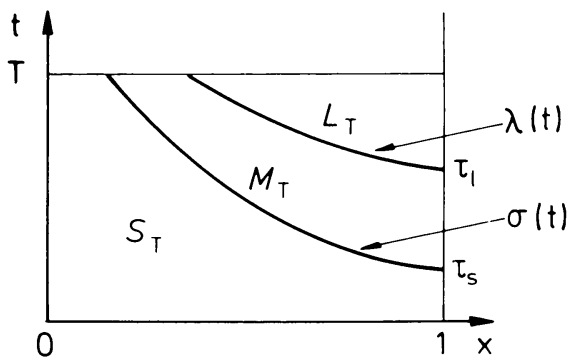

Figure 2. The sets $\mathscr{S}_{T}, \mathscr{L}_{T}$ and $\mathscr{M}_{T}$

Definition 1.2. A triple $(u, \sigma, \lambda)$ is a classical subsolution of Problem I on $[0, T]$ if (i) $u \in C^{2,1}\left(\overline{\mathscr{S}}_{T} \cup \overline{\mathscr{L}}_{T}\right), \sigma \in C^{1}\left(\left(\tau_{s}, T\right]\right) \cap C\left(\left[\tau_{s}, T\right]\right), \lambda \in C^{1}\left(\left(\tau_{l}, T\right]\right) \cap C\left(\left[\tau_{l}, T\right]\right)$ and the limits

$$
u(\sigma(t)+, t):=\lim _{x \downarrow \sigma(t)} u(x, t), \quad u(\lambda(t)-, t)=\lim _{x \uparrow \lambda(t)} u(x, t)
$$

exist for all $t \in\left(\tau_{s}, T\right]$, respectively, $t \in\left(\tau_{l}, T\right]$;

(ii) $(\phi(u))_{x x}+f-u_{t} \geqslant 0$ in $\mathscr{S}_{T} \cup \mathscr{L}_{T}, f-u_{t} \geqslant 0$ in $\mathscr{M}_{T}$;

(iii) $u \leqslant u_{0}$ a.e. in $\bar{\Omega} \times\{0\}$;

(iv) $u(0, t) \leqslant 0,(\phi(u))_{x}(1, t) \leqslant 0$ a.e. on $[0, T]$;

(v) $u(\sigma(t)-, t)=\alpha$ for $t \in\left[\tau_{s}, T\right], u(\lambda(t)+, t)=\beta$ for $t \in\left[\tau_{l}, T\right]$;

(vi)

$$
\begin{array}{ll}
(\phi(u))_{x}(\sigma(t)-, t) \leqslant \sigma^{\prime}(t)[u(\sigma(t)+, t)-\alpha], & t \in\left(\tau_{s}, T\right], \\
(\phi(u))_{x}(\lambda(t)+, t) \geqslant \lambda^{\prime}(t)[u(\lambda(t)-, t)-\beta], & t \in(\tau, T] .
\end{array}
$$

A supersolution is defined likewise, but with the inequalities reversed, and a solution is defined by replacing the inequalities by equalities.

As in [3] we can prove that classical sub- and supersolutions are also weak suband supersolutions. 
The existence, uniqueness and regularity of solutions of Problem I are well established $[1,3]$. For convenience we summarize the main results in $\S 2$. Some results concerning stationary solutions of Problem I are derived there as well.

In $\$ 3$ we prove that the mushy region and the liquid region both appear in finite time, and that the boundary of $\mathscr{M}$ consists of continuous curves. The continuity of $u$ near the solid/mush interface will also be discussed in this section.

In $\$ 4$ we prove that after a finite time $T^{*}$, the mushy region ceases to exist, i.e., that $\mathscr{M}(t)=\varnothing$ for all $t \geqslant T^{*}$.

2. Basic properties of the solutions. We begin with a standard existence and uniqueness theorem.

TheOREM 2.1. Suppose hypothesis $\mathrm{H}$ is satisfied. Then for any $T>0$, Problem I has a unique weak solution $u(t)$ on $[0, T]$. There exists a constant $K>0$, independent of $T$, such that $0 \leqslant u(t) \leqslant K$ for all $t \in[0, T]$.

The proof can be given by a standard regularization procedure (see for instance [ 1 and 3]).

A central role will be played by the following Comparison Principle for (weak) sub- and supersolutions. For the proof we also refer to [1].

THEOREM 2.2. Let hypothesis $\mathrm{H}$ be satisfied, and let $u(t)$ be a (weak) subsolution and $\hat{u}(t) a$ (weak) supersolution of Problem $\mathrm{I}$ on $[0, T]$, with initial values $u_{0}$ and $\hat{u}_{0}$, respectively. If $u_{0} \leqslant \hat{u}_{0}$ a.e. in $\Omega$, then $u(t) \leqslant \hat{u}(t)$ a.e. in $\Omega$ for all $t \in[0, T]$.

Theorem 2.2 and the assumption that $u_{0}$ is a subsolution of Problem I allow us to deduce the following monotonicity and convergence theorem for solutions $u(t)$ of Problem I.

TheOREM 2.3. Let hypothesis $\mathrm{H}$ be satisfied, and let $u(t)$ be the solution of Problem I on $[0, T]$. Then

(i) $t \rightarrow u(t)$ is nondecreasing a.e. in $\Omega$;

(ii) for any $t>0,(\phi(u))_{x}(t) \in L^{\infty}(\Omega)$ and $\phi(u) \in C^{\nu, \nu / 2}\left(\bar{Q}_{T}\right)$ for every $\nu \in(0,1)$;

(iii) $\phi(u)(t)$ converges uniformly as $t \rightarrow \infty$ to $\phi(w)$, where $w$ is the stationary solution of Problem I defined in $\mathrm{H} 4$.

Proof. Part (i) is standard. To prove part (ii) we observe that $u_{t}(t)$ is a positive distribution, and hence a positive measure. Thus $(\phi(u))_{x x}$ is a measure which is bounded below, whence $(\phi(u))_{x}(t) \in L^{\infty}(\Omega)$. The Hölder continuity of $\phi(u)$ in $\bar{Q}_{T}$ follows from Sobolev's embedding theorem and a lemma due to Gilding [5]. (iii) By the monotonicity proved in part (i) and the boundedness implied by $\mathrm{H} 4$, there exists a function $u_{\infty} \in L^{\infty}(\Omega)$ such that

$$
u(t) \rightarrow u_{\infty} \quad \text { as } t \rightarrow \infty \text { in } L^{p}(\Omega)
$$

for any $p>1$. Likewise we have

$$
f(u)(t) \rightarrow f\left(u_{\infty}\right) \text { as } t \rightarrow \infty \text { in } L^{p}(\Omega)
$$

for any $p>1$. 
By part (ii) $\phi(u)(1) \in W^{1,2}(\Omega)$ and hence, following [3], $\{\phi(u)(t): t \geqslant 1\}$ is bounded in $W^{1,2}(\Omega)$, whence there exists a function $V_{\infty} \in C(\bar{\Omega})$ such that

$$
\phi(u)(t) \rightarrow v_{\infty} \quad \text { as } t \rightarrow \infty \text { in } C(\bar{\Omega}) .
$$

By the continuity of $\phi$, it follows that $v_{\infty}=\phi\left(u_{\infty}\right)$.

Thus it remains to prove that $u_{\infty}=w$. To do this we take a test function $\psi \in C^{2}(\bar{\Omega})$ such that $\psi \geqslant 0$ in $\Omega, \psi(0)=0$ and $\psi^{\prime}(1)=0$. In view of the definition of a solution we have

$$
\int_{\Omega} u(t) \psi-\int_{\Omega} u_{0} \psi=\int_{0}^{t} \int_{\Omega}\left\{\phi(u) \psi_{x x}+f(u) \psi\right\} .
$$

If we divide by $t$, let $t \rightarrow \infty$ and use the convergence properties of the functions $u(t), \phi(u)(t)$ and $f(u)(t)$, we obtain

$$
\int_{\Omega}\left\{\phi\left(u_{\infty}\right) \psi_{x x}+f\left(u_{\infty}\right) \psi\right\}=0 .
$$

Thus, $u_{\infty}$ is a (weak) stationary solution of Problem I.

We conclude this section by noting the following properties of stationary solutions of Problem I.

THEOREM 2.4. Suppose hypothesis $\mathrm{H}$ is satisfied, and let $w$ be a stationary solution of Problem I such that $\phi(w)(1)>1$. Then

(i) $\phi(w) \in C^{1, \gamma}(\bar{\Omega})$ for any $\gamma \in(0,1)$;

(ii) the set $\mathscr{M}^{*}:=\{x \in \Omega: \phi(w)(x)=1\}$ consists of a single point $\left\{a^{*}\right\}$, and $0<a^{*}<1$;

(iii) $\phi(w) \in C^{2}\left(\Omega \backslash\left\{a^{*}\right\}\right), \quad w \in C\left(\bar{\Omega} \backslash\left\{a^{*}\right\}\right)$ and $\lim _{x \uparrow a^{*}} w(x)$ exists, $\lim _{x \downarrow a^{*}} w(x)$ exists;

(iv) $(\phi(w))^{\prime \prime}+f(w)=0$ in $\Omega \backslash\left\{a^{*}\right\}$;

(v) $\phi(w)(0)=(\phi(w))^{\prime}(1)=0$ and $w\left(a^{*}-\right)=\alpha, w\left(a^{*}+\right)=\beta$.

Proof. Property (i) results immediately from the fact that $(\phi(w))^{\prime \prime}+f(w)=0$ holds in the sense of $\mathscr{D}^{\prime}(\Omega)$ and that $f(w) \in L^{\infty}(\Omega)$. To prove (ii) observe that by the maximum principle, $\phi(w)$ cannot have any positive minimum in $\Omega$. Thus, the level sets of $\phi(w)$ are intervals. To show that $\mathscr{M}^{*}$ consists of a single point, it suffices to show that $\mathscr{\mathscr { M }}^{*}=\varnothing$. Suppose not. Then for any test function $\psi$ with support $K \subset \mathscr{\mathscr { M }}^{*}$,

$$
0=\int_{K}\left\{\phi(w) \psi^{\prime \prime}+f(w) \psi\right\}=\int_{K} f(w) \psi,
$$

whence $f(w)=0$ a.e. in $\mathscr{M}^{*}$, which contradicts $\mathrm{H} 4$.

The remaining properties follow from the equation being satisfied in $\mathscr{D}^{\prime}(\Omega)$ and the existence of a continuous inverse of $\phi$ on $\mathbf{R}^{+} \backslash(\alpha, \beta)$.

3. Continuity properties of the interfaces. In this section we establish the continuity of the boundary of the set $\mathscr{M}$ and the continuity of $u$ across the (receding) solid/mush interface $\sigma$.

To begin with we note the following monotonicity property of the solution of Problem I. 
Lemma 3.1. Let hypothesis $\mathrm{H}$ be satisfied. Then for any $t>0, \phi(u)(t)$ is $a$ nondecreasing function on $[0,1]$.

Proof. This follows by regularizing the equation and applying the maximum principle to the spatial derivative of the regularized solution, and finally passing to the limit.

As a consequence of Lemma 3.1, $\phi(u)(t)$ attains its maximum value at $x=1$, and its level sets are intervals of the form $[l(t), 1], 0 \leqslant l(t) \leqslant 1$.

Let us write

$$
\begin{gathered}
\mathscr{S}(t):=\{x \in \bar{\Omega}: \phi(u)(x, t)<1\}, \quad \mathscr{L}(t):=\{x \in \bar{\Omega}: \phi(u)(x, t)>1\}, \\
\mathscr{M}(t):=\{x \in \bar{\Omega}: \phi(u)(x, t)=1\}
\end{gathered}
$$

and let

$$
\begin{gathered}
T_{S}:=\inf \{t \geqslant 0: \mathscr{M}(t) \neq \varnothing\}, \quad T_{L}:=\inf \{t \geqslant 0: \mathscr{L}(t) \neq \varnothing\}, \\
\sigma(t):=\min \{x \in \mathscr{M}(t)\}, \quad t \geqslant T_{S}, \quad \lambda(t):=\max \{x \in \mathscr{M}(t)\}, \quad t \geqslant T_{L} .
\end{gathered}
$$

By Theorem 2.3,u(t) is nondecreasing on $[0, T]$ so that $T_{S} \leqslant T_{L}$. Thus, using Lemma 3.1 we can write

$$
\begin{aligned}
\mathscr{M}_{T}= & \left\{(x, t) \in \bar{Q}_{T}: \sigma(t) \leqslant x \leqslant 1, T_{S} \leqslant t \leqslant T_{L}\right\} \\
& \cup\left\{(x, t) \in \bar{Q}_{T}: \sigma(t) \leqslant x \leqslant \lambda(t), T_{L}<t \leqslant T\right\} .
\end{aligned}
$$

Lemma 3.2. Assume hypothesis $\mathrm{H}$ is satisfied. Then

(i) $0<T_{S}<\infty$;

(ii) $\sigma(t)$ is strictly decreasing and $\sigma\left(T_{S}\right)=1$;

(iii) $\sigma \in C\left(\left[T_{S}, T\right]\right)$ for any $T>T_{S}$.

Let $w$ be the stationary solution defined in $\mathrm{H} 4$, and let $a^{*}$ be defined by $\phi\left(w\left(a^{*}\right)\right)=1$. Then

(iv) $\sigma(t) \rightarrow a^{*}$ as $t \rightarrow \infty$.

Proof. (i) By $\mathrm{H} 3$ and the continuity of $\phi(u)$ in $Q, T_{S}>0$. If $T_{S}=\infty, \phi(u)(t)<1$ for all $t>0$, which contradicts the convergence of $u(t)$ to $w$ established in Theorem 2.3.

(ii) By Lemma 3.1, $\sigma$ is nonincreasing. The strict monotonicity is proved as in [3, Theorem 4.1(ii)].

(iii) Suppose $\sigma$ is not continuous. Then there exists a time $t_{0}>T_{S}$ such that

$$
\lim _{t \downarrow t_{0}} \sigma(t)=x_{0}<x_{1}=\lim _{t \uparrow t_{0}} \sigma(t) .
$$

Consider the rectangle $R=\left(x_{0}, x_{1}\right) \times\left(0, t_{0}\right]$. Then $u \leqslant \alpha$ on the parabolic boundary of $R$ and $u=\alpha$ on the top: $\left[x_{0}, x_{1}\right] \times\left\{t_{0}\right\}$. Because $\phi^{\prime}\left(\alpha^{-}\right)>0$, we may apply the strong maximum principle to deduce that $u \equiv \alpha$ throughout $\bar{R}$. Since $u_{0}<\alpha$, this is not possible, whence $\sigma$ must be continuous.

(iv) That $\sigma(t)$ tends to a limit follows from part (ii). That the limit is $a^{*}$ may be deduced from Theorem 2.3. 
Corresponding properties can be proved for the interface $\lambda$. Their proofs are very similar to those for $\sigma$, except for the continuity, which needs further preparation. Thus we state without proof

LEMMA 3.3. Under hypothesis $\mathrm{H}$ we have

(i) $T_{S} \leqslant T_{L}<\infty$;

(ii) $\lambda(t)$ is strictly decreasing and $\lambda\left(T_{L}\right)=1$;

(iii) $\lambda(t) \rightarrow a^{*}$ as $t \rightarrow \infty$.

Before proving the continuity of $\lambda$, we shall establish the continuity of $u$ across the interface $\sigma$.

Lemma 3.4. Suppose hypothesis $\mathrm{H}$ is satisfied. Then

(i) $T_{S}<T_{L}$;

(ii) let $t_{0} \geqslant T_{S}$ and let $\sigma\left(t_{0}\right)<\lambda\left(t_{0}\right)$ if $t_{0}>T_{L}$.

Then for any $\varepsilon>0$ there exist positive constants $\delta$ and $\tau$ such that

$$
\underset{R_{0}}{\text { ess sup }}\left|u(x, t)-u\left(x, t_{0}\right)\right|<\varepsilon
$$

where $R_{0}=\left(0, \sigma\left(t_{0}\right)+\delta\right) \times\left(t_{0}-\tau, t_{0}+\tau\right)$.

Proof. Write $x_{0}:=\sigma\left(t_{0}\right)$ and define

$$
x_{1}:= \begin{cases}\frac{1}{2}\left\{x_{0}+\lambda\left(t_{0}\right)\right\} & \text { if } t_{0}>T_{L}, \\ \frac{1}{2}\left(x_{0}+1\right) & \text { otherwise. }\end{cases}
$$

Then, if $t_{0}>T_{S}, x_{0}<x_{1}<1$, if $t_{0}=T_{S}, x_{0}=x_{1}=1$ and

$$
m:=\underset{\left[x_{0}, x_{1}\right]}{\operatorname{ess} \sup } u\left(x, t_{0}\right)<\beta .
$$

We claim that for any $\varepsilon>0$ there exists a constant $\tau_{\varepsilon}>0$ such that

$$
u(x, t) \leqslant \alpha+\varepsilon \quad \text { for }(x, t) \in\left[0, x_{0}\right] \times\left[t_{0}, t_{0}+\tau_{\varepsilon}\right] .
$$

Let us prove (3.1). Since $\phi(u)\left(x, t_{0}\right)<1$ for all $x \in\left(0, x_{0}\right)$, we can choose constants $A \in(0,1)$ and $\eta \in\left(0, x_{0}\right)$ such that

$$
A+\frac{1-A}{x_{0}-\eta} x>\phi(u)\left(x, t_{0}\right) \text { in }\left[0, x_{0}\right]
$$

If $t_{0}>T_{S}$, and thus $x_{1}<1$, we can also find a constant $B>0$ such that

$$
1+B\left(x-x_{1}\right)>\phi(u)\left(x, t_{0}\right) \text { in }\left[x_{1}, 1\right] \text {. }
$$

Introducing the quantities

$$
\begin{gathered}
\gamma:=\sup \phi^{\prime} ; \quad K:=\max \left\{\max f, m, \frac{B^{2}}{\gamma(\beta-\alpha)}\right\} ; \\
\tilde{\sigma}(t):=\left(x_{0}-\eta\right)\left\{1-\frac{\gamma K}{1-A}\left(t-t_{0}\right)\right\} ; \tilde{\lambda}(t):=x_{1}-\frac{\gamma K}{B}\left(t-t_{0}\right) ; \\
\tau_{\varepsilon}^{\prime}:=\frac{1}{K} \min \{\varepsilon, \beta-m\} \quad \text { and } \tau_{\varepsilon}:= \begin{cases}\tau_{\varepsilon}^{\prime} & \text { if } t_{0}>T_{S}, \\
\min \left\{\tau_{\varepsilon}^{\prime}, \frac{x_{1}-x_{0}}{\gamma} B\right\} & \text { if }\end{cases}
\end{gathered}
$$


we define for $t \in\left[t_{0}, t_{0}+\tau_{\varepsilon}\right)$ the function $\tilde{u}$ :

$$
\tilde{u}(x, t):= \begin{cases}\phi^{-1}\left(A+\frac{1-A}{x_{0}-\eta} x+\gamma K\left(t-t_{0}\right)\right), & 0 \leqslant x \leqslant \tilde{\sigma}(t), \\ \alpha+K\left(t-t_{0}\right), & \tilde{\sigma}(t) \leqslant x \leqslant x_{0}\end{cases}
$$

and, in the case $t_{0}>T_{S}$,

$$
\tilde{u}(x, t):= \begin{cases}m+K\left(t-t_{0}\right), & x_{0}<x \leqslant \tilde{\lambda}(t), \\ \phi^{-1}\left(1+B\left(x-x_{1}\right)+\gamma K\left(t-t_{0}\right)\right), & \tilde{\lambda}(t)<x \leqslant 1 .\end{cases}
$$

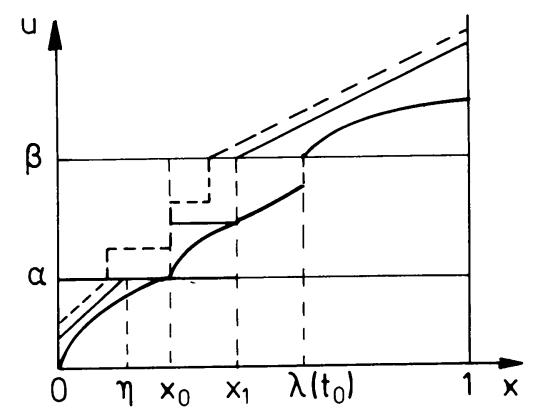

FIGURE 3. The function $\tilde{u}$ in case $\phi$ is piecewise linear

A straightforward calculation shows that $\tilde{u}$ is a classical supersolution of Problem I on $\bar{\Omega} \times\left[t_{0}, t_{0}+\tau_{\varepsilon}\right]$. Thus by the comparision principle, $\tilde{u}(x, t) \geqslant u(x, t)$ a.e. in $\Omega \times\left[t_{0}, t_{0}+\tau_{\varepsilon}\right]$, whence (3.1) follows.

By putting $t_{0}=T_{S}$ in (3.1) we find that $T_{L}>T_{S}$.

Combining the monotonicity of $t \rightarrow u(x, t)$ and the continuity of $\sigma$ with (3.1), the desired continuity property of $u$ follows.

It so happens that we can actually compute the time interval $T_{L}-T_{S}$. This will be done in

LEMMA 3.5. Under hypothesis $\mathrm{H}$ we have

$$
T_{L}-T_{S}=\int_{\alpha}^{\beta} \frac{d s}{f(s)} .
$$

More generally, for $t>T_{S}$,

$$
t-\sigma^{-1}(x)=\int_{\alpha}^{u(x, t)} \frac{d s}{f(s)}
$$

holds a.e. on $\mathscr{M}(t)=(\sigma(t), \tilde{\lambda}(t))$, where $\tilde{\lambda}(t)=1$ if $T_{S}<t \leqslant T_{L}$ and $\tilde{\lambda}(t)=\lambda(t)$ if $t>T_{L}$.

Proof. As in [3, Theorem 3.4] we find that

$$
u(x, t)-u(x, s)=\int_{s}^{t} f(u)(x, \tau) d \tau \quad \text { a.e. in } \mathscr{M}(t)
$$


when $s>\sigma^{-1}(x)$, or

$$
t-t_{0}=\int_{u\left(x, t_{0}\right)}^{u(x, t)} \frac{d s}{f(s)} \text { a.e. in } \mathscr{M}(t),
$$

provided $t_{0}>\sigma^{-1}(x)$. Thus, letting $t_{0} \downarrow \sigma^{-1}(x)$ and using the continuity of $u$ near $\sigma$ proved in Lemma 3.4, we obtain

$$
t-\sigma^{-1}(x)=\int_{\alpha}^{u(x, t)} \frac{d s}{f(s)} \text { a.e. in } \mathscr{M}(t) .
$$

This proves (3.3). If we now set $t=T_{L}$ and let $x \rightarrow 1$ through a sequence on which (3.3) holds, we obtain

$$
T_{L}-T_{S}=\int_{\alpha}^{\bar{u}} \frac{d s}{f(s)},
$$

where $\bar{u}=\lim _{x \rightarrow 1} u\left(x, T_{L}\right)$. Clearly $\bar{u} \leqslant \beta$. However, if $\bar{u}<\beta$, then $u \leqslant \bar{u}<\beta$ in $\bar{\Omega} \times\left[0, T_{L}\right]$. By using a comparision function similar to the one constructed in the proof of Lemma 3.4 (for $x_{0}=1$ ), we can deduce that this implies that $u\left(1, T_{L}\right)<\beta$, which contradicts the definition of $T_{L}$. Thus $\bar{u}=\beta$, and the proof of (3.2) is complete.

We conclude this section by proving the continuity of the mush/liquid interface $\lambda$.

LEMMA 3.6. Under hypothesis $\mathrm{H}$ the interface curve $\lambda$ is continuous at all $t \geqslant T_{L}$ where $\lambda(t)>\sigma(t)$.

Proof. Because of the monotonicity of $\lambda$ and $u$, it is sufficient to prove that if $\lambda\left(t_{0}\right)>\sigma\left(t_{0}\right)$, then for any small $\varepsilon>0$, there exists a time $\tau_{\varepsilon}>0$ such that

$$
\lambda(t)>\lambda\left(t_{0}\right)-2 \varepsilon \text { for } t_{0} \leqslant t<t_{0}+\tau_{\varepsilon} .
$$

To this end, let

$$
x_{0}=\lambda\left(t_{0}\right) \Rightarrow u\left(x_{0}, t_{0}\right) \leqslant \beta .
$$

By the strict monotonicity of $t \rightarrow u(t)$ (cf. Lemma 3.5) we have

$$
u\left(x, t_{0}\right)<\beta \quad \text { a.e. in }\left[0, x_{0}\right) \text {. }
$$

Let $\varepsilon>0$ be a small number and define

$$
\delta(\varepsilon):=\beta-\operatorname{ess} \sup \left\{u\left(x, t_{0}\right): x \in\left[0, x_{0}-\varepsilon / 2\right]\right\} .
$$

By (3.5), $\delta(\varepsilon)>0$ and without loss of generality we may suppose that $\delta(\varepsilon)<\beta-\alpha$. We define the following quantities: $L>0$ to be such that

$$
\begin{gathered}
\phi(u)\left(x, t_{0}\right) \leqslant 1+L\left(x-x_{0}+\varepsilon\right) \quad \text { when } x \in\left[x_{0}-\varepsilon, 1\right], \\
M:=\max \left\{\sup f, 2 L^{2} / \gamma \delta(\varepsilon)\right\}, \quad \hat{\tau}=L^{2} / \gamma M^{2}, \\
\hat{\lambda}(t)=x_{0}-\varepsilon-(\gamma M / L)\left(t-t_{0}\right), \quad t \in\left[t_{0}, t_{0}+\tau\right],
\end{gathered}
$$

where $\gamma=\sup \phi^{\prime}$ has been defined before. For $t \in\left[t_{0}, t_{0}+\hat{\tau}\right]$ we define the function

$$
\hat{u}(x, t)= \begin{cases}\phi^{-1}\left(1+L\left(x-x_{0}+\varepsilon\right)+\gamma M\left(t-t_{0}\right)\right), & x \in[\hat{\lambda}(t), 1], \\ \beta-\delta(\varepsilon)+M\left(t-t_{0}\right), & x \in[0, \hat{\lambda}(t)) .\end{cases}
$$


An elementary calculation confirms that $\hat{u}$ is a supersolution of Problem I on $\bar{\Omega} \times\left[t_{0}, t_{0}+\tau\right]$. Thus, the comparision theorem implies that $\hat{u}(t) \geqslant u(t)$ for $t \in$ $\left[t_{0}, t_{0}+\hat{\tau}\right]$ and hence

$$
\hat{\lambda}(t) \leqslant \lambda(t) \text { for } t \in\left[t_{0}, t_{0}+\hat{\tau}\right]
$$

Remembering the definition of $\hat{\lambda}$ (3.7) and introducing $\tau=\min \{\hat{\tau}, \varepsilon L /(\gamma M)\}$, we obtain (3.4).

4. Extinction of the mushy region in finite time. In this section we prove that there is a (finite) time $T^{*}>T_{L}$ such that $\mathscr{M}\left(T^{*}\right)=\varnothing$.

We begin with a technical lemma, which is proved in the Appendix.

LEMMA 4.1. Let $g$ be a continuous function on $[0, \infty)$ such that $g(0)>0$, and let $y$ : $[0, T] \rightarrow \mathbf{R}$ satisfy $y^{\prime}=c g(y)-m t, y(0)=0$, in which $c, m \in \mathbf{R}^{+}$. Then for any $t_{0} \in[0, T], y_{0} \in \mathbf{R}^{+}$, there exists a constant $c>0$ such that, for some $\tau \in\left(0, t_{0}\right)$ and $\eta \in\left(0, y_{0}\right), y(\tau)=\eta, y^{\prime}(\tau)=0$.

Recall that $w$ is the minimal solution of Problem II such that $w \geqslant u_{0}$ in $\Omega$, defined on $\mathrm{H} 4$.

Lemma 4.2. Let hypothesis $\mathrm{H}$ be satisfied. Suppose $\bar{\beta} \in(\beta, w(1))$ and $\bar{t} \in\left(T_{I}, \infty\right)$ are so chosen that $u(1, \bar{t})=\bar{\beta}$ and $\sigma(\bar{t})<\lambda(\bar{t})<1$. Then there exists a function $\bar{u}$ which, for $t \geqslant \bar{t}$ has all the properties of a classical subsolution except the boundary condition at $x=0$. Its mush/liquid interface $\bar{\lambda}(t)$ is given by $\bar{\lambda}(t)=1-a+c(t-\bar{t})$ where $a \in(\lambda(\bar{t}), 1)$ and $c>0$ are suitable constants, and $\bar{u}(x, \bar{t}) \leqslant u(x, \bar{t})$ a.e. in $[\sigma(\bar{t}), 1]$.

REMARK. The existence of constants $\bar{\beta}$ and $\bar{t}$ with the stated properties follows from the results of $\S 3$.

Proof. We construct a family of travelling wave solutions $q=q(x+c t), c>0$, of the differential equation in Problem $\mathrm{I}$, in which the source term $f$ is replaced by a constant $m \in \mathbf{R}^{+}$, where $m=\min \{f(s): \alpha \leqslant s \leqslant w(1)\}$.

Substitution yields the equation

$$
(\phi(q))^{\prime \prime}-c q^{\prime}+m=0
$$

in which primes denote differentiation with respect to the variable $\xi=x+c t$. Integrating (4.1) once we obtain

$$
(\phi(q))^{\prime}-c q+m \xi=C,
$$

where $C$ is a constant. Fixing the interface $q=\beta$ at $\xi=0$, and prescribing that $q$ jump there from $\beta$ to $\alpha$, we find, in view of the interface condition

$$
(\phi(q))^{\prime}(0+)=c(\beta-\alpha),
$$

and hence, by (4.2), $C=-c \alpha$. Putting $p=\phi(q)$ and $q=\eta(p)$, for $q \geqslant \beta$, we get for (4.2) that

$$
p^{\prime}=c\{\eta(p)-\alpha\}-m \xi, \quad p(0)=1 .
$$

Since $\phi^{\prime}\left(\beta^{+}\right)>0$, the right side of the differential equation is locally Lipschitz continuous on $[0, \infty)$, whence the local existence and uniqueness of a solution is ensured. 
The assumptions of Lemma 4.1 being fulfilled, we conclude that there exists a constant $c>0$ such that the solution $p$ of (4.3) satisfies $p(a)=\phi(\hat{\beta})$ and $p^{\prime}(a)=0$ for some $a \in(0,1-\sigma(\bar{t}))$ and $\hat{\beta} \in(\beta, \bar{\beta})$.

Having chosen $c$ in such a way, let

$$
t^{*}=\max \left\{\frac{1-a}{c}, \sup \left\{t>T_{L}, \sigma(t)<\lambda(t)\right\}\right\},
$$

and

$$
\bar{\lambda}(t)=1-a+c(t-\bar{t}), \quad t \in\left[\bar{t}, t^{*}\right] .
$$

For $t \in\left[\bar{t}, t^{*}\right]$ define

$$
\bar{u}(x, t)= \begin{cases}\alpha, & 0 \leqslant x<\bar{\lambda}(t), \\ q(x+c(t-\bar{t})-1+\alpha), & \bar{\lambda}(t) \leqslant x \leqslant \bar{\lambda}(t)+a, \\ \hat{\beta}, & \bar{\lambda}(t)+a<x \leqslant 1 .\end{cases}
$$

It is easy to verify that $\bar{u}$ meets all the requirements for a classical subsolution except the boundary condition at $x=0$.

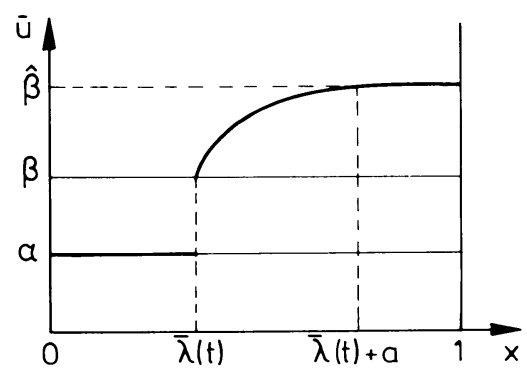

Figure 4. The function $\bar{u}$

We are now ready to prove the main result: the extinction of the mushy region $\mathscr{M}$ in finite time.

THEOREM 4.3. Suppose hypothesis $\mathrm{H}$ is satisfied. Then there exists a time $T^{*} \in$ $\left(T_{L}, \infty\right)$ such that $\sigma\left(T^{*}\right)=\lambda\left(T^{*}\right)$.

Proof. We assume the assertion is false-i.e., $\sigma(t)<\lambda(t)$ for all $t>T_{L}$-and show that this leads to a contradiction.

Choose $\bar{t} \geqslant T_{L}, \bar{\beta} \in(\beta, w(1))$ and select $\hat{\beta} \in(\beta, \bar{\beta}), a \in(\lambda(\bar{t}), 1)$ and $c>0$ as in Lemma 4.2. Let $\bar{\lambda}(t)$ be the interface curve (4.4) associated with $\bar{u}$, defined for $t \in[\bar{t}, \bar{t}+(1-a) / c]$. Since $\bar{\lambda}(\bar{t}+(1-a) / c)=0$, there must exist a time $\tilde{t} \in$ $[\bar{t}, \bar{t}+(1-a) / c)$ such that

$$
\bar{\lambda}(\tilde{t})=\sigma(\tilde{t}) .
$$

Define a continuous, decreasing function $\zeta:[\bar{t}, \tilde{t}] \rightarrow(0,1)$ such that

$$
\sigma(t)<\zeta(t)<\min \{\lambda(t), \bar{\lambda}(t)\} \text { for } \bar{t} \leqslant t<\tilde{t} .
$$


Fix $\hat{t} \in[\bar{t}, \tilde{t})$ arbitrarily and partition the set $Q_{i, \hat{t}}:=\{x \in \Omega: \bar{t}<t \leqslant \hat{t}\}$ as $\bar{Q}_{i, \hat{i}}=P^{-} \cup P^{+}$, where

$$
P^{-}:=\left\{(x, t) \in \bar{Q}_{i, \hat{i}}: 0 \leqslant x \leqslant \zeta(t)\right\}, \quad P^{+}:=\left\{(x, t) \in \bar{Q}_{i, \hat{i}}: \zeta(t)<x \leqslant 1\right\} .
$$

On $\bar{Q}_{i, \hat{i}}$ we now define the function

$$
U= \begin{cases}u & \text { in } P^{-} \\ \bar{u} & \text { in } P^{+}\end{cases}
$$

We prove that this function is a (weak) subsolution of Problem I.

We introduce a further partition of $\bar{Q}_{i, \hat{i}}$. To this end we define continuous decreasing functions $\sigma_{1}, \sigma_{2}, \lambda_{1}, \lambda_{2}:[\bar{t}, \hat{t}] \rightarrow(0,1)$, such that

$$
\sigma<\sigma_{1}<\sigma_{2}<\zeta<\lambda_{2}<\lambda_{1}<\lambda \text { on }[\bar{t}, \hat{t}]
$$

and subsets of $\bar{Q}_{i, \hat{i}}$ :

$$
\begin{gathered}
\Sigma_{1}=\left\{0 \leqslant x \leqslant \sigma_{1}(t)\right\}, \quad \Sigma_{2}=\left\{\sigma_{1}(t)<x<\sigma_{2}(t)\right\}, \\
Z=\left\{\sigma_{2}(t) \leqslant x \leqslant \lambda_{2}(t)\right\}, \\
\Lambda_{2}=\left\{\lambda_{2}(t)<x<\lambda_{1}(t)\right\}, \quad \Lambda_{1}=\left\{\lambda_{1}(t) \leqslant x \leqslant 1\right\} .
\end{gathered}
$$

Then $\bar{Q}_{i, i}=\Sigma_{1} \cup \Sigma_{2} \cup Z \cup \Lambda_{2} \cup \Lambda_{1}$.

Define three functions $\eta_{i} \in C^{\infty}\left(\bar{Q}_{i, \hat{i}}\right)$ such that

$$
\begin{gathered}
\eta_{1}=\left\{\begin{array}{ll}
1 & \text { on } \Sigma_{1}, \\
0 & \text { on } Z \cup \Lambda_{2} \cup \Lambda_{1},
\end{array} \quad \eta_{2}= \begin{cases}0 & \text { on } \Sigma_{1} \cup \Sigma_{2} \cup Z, \\
1 & \text { on } \Lambda_{1},\end{cases} \right. \\
\eta_{3}= \begin{cases}1 & \text { on } Z, \\
0 & \text { on } \Sigma_{1} \cup \Lambda_{1},\end{cases}
\end{gathered}
$$

and $0 \leqslant \eta_{i} \leqslant 1(i=1,2,3), \eta_{1}+\eta_{2}+\eta_{3}=1$. Then any test function $\psi$ can be written as $\psi=\psi_{1}+\psi_{2}+\psi_{3}$, where $\psi_{i}=\psi \eta_{i}$. Clearly the functions $\psi_{i}$ are all admissible test functions for Problem I.

Let $t \in[\bar{t}, \hat{t}]$ and consider for any test function $\psi$ the quantity

$$
q[\psi]=\int_{\Omega} U(t) \psi(t)-\int_{\Omega} U(\bar{t}) \psi(\bar{t})-\iint_{\bar{Q}_{i, i}}\left[U \psi_{t}+\phi(U) \psi_{x x}+f(U) \psi\right] .
$$

To prove that $U$ is a subsolution we need to show that $q[\psi] \leqslant 0$ for any $\psi$.

Write

$$
q[\psi]=q\left[\psi_{1}\right]+q\left[\psi_{2}\right]+q\left[\psi_{3}\right]
$$

Since in $q\left[\psi_{1}\right]$ the integrals are taken over parts of $\Sigma_{1} \cup \Sigma_{2}$, we can replace $U$ in this expression by $u$, whence

$$
q\left[\psi_{1}\right]=0
$$

Similarly, we can replace $U$ in $q\left[\psi_{2}\right]$ by $\bar{u}$. By Lemma 4.2 and the fact that $\psi_{2}$ vanishes near $x=0$, it follows that

$$
q\left[\psi_{2}\right] \leqslant 0
$$


Finally, consider $q\left[\psi_{3}\right]$. Since the integrals involved are taken over a subset of $\mathscr{M}$, $\bar{u}=\alpha$ in $\mathscr{M}$ and $\psi_{3}$ vanishes near the boundary of $\Sigma_{2} \cup Z \cup \Lambda_{2}$, an easy calculation shows that

$$
q\left[\psi_{3}\right]=\int_{i}^{t} \int_{\zeta}^{\lambda_{1}}[m-f(\alpha)] \psi_{3}+\hat{q}\left[\psi_{3}\right] \leqslant \hat{q}\left[\psi_{3}\right]
$$

where

$$
\begin{aligned}
\hat{q}\left[\psi_{3}\right]:= & \int_{\sigma_{1}(t)}^{\zeta(t)} u(t) \psi_{3}(t)-\int_{\sigma_{1}(\bar{t})}^{\zeta(\bar{t})} u(\bar{t}) \psi_{3}(\bar{t}) \\
& -\int_{\bar{i}}^{t} \int_{\sigma_{1}}^{\zeta}\left[u \psi_{3 t}+f(u) \psi_{3}\right] .
\end{aligned}
$$

Since $\tau$ and $\lambda$ are monotonic and continuous, it is possible to choose $\zeta$ to be almost everywhere differentiable. Since the domain of integration in (4.10) is contained in $\mathscr{M}$, we can proceed as in Lemma 3.5 , to obtain

$$
\hat{q}\left[\psi_{3}\right]=\int_{i}^{t} \zeta^{\prime} u(\zeta(\tau), \tau) \psi_{3}(\zeta(\tau), \tau) d \tau \leqslant 0
$$

since $\zeta$ is decreasing.

Thus we have proved that $U$ is a subsolution of Problem I. This means that $U \leqslant u$ a.e. in $\bar{Q}_{i, \hat{i}}$ and, in particular, that $\bar{\lambda}(t) \geqslant \lambda(t)$ for $t \in[\bar{t}, \hat{t}]$. Since $\hat{t}$ was chosen arbitrarily in $[\bar{t}, \hat{t}]$ we may conclude, in view of the continuity of $\lambda$, that $\bar{\lambda}(\tilde{t}) \geqslant \lambda(\tilde{t})$. But we had chosen $\tilde{t}$ so that $\bar{\lambda}(\tilde{t})=\sigma(\tilde{t})$, whence we find that $\sigma(\tilde{t}) \geqslant \lambda(\tilde{t})$, which contradicts our original assumption that $\sigma(t)<\lambda(t)$ for all $t>T_{L}$. This completes the proof.

\section{Appendix.}

Proof of Lemma 4.1. Define $B:=\max \left\{g(y): 0 \leqslant y \leqslant y_{0}\right\}$ and let $s=\sup \{t>0$ : $y<y_{0}$ on $\left.(0, t)\right\}$. Then, by the differential inequality

$$
y^{\prime} \leqslant c B-m t \quad \text { for } 0 \leqslant t \leqslant s
$$

we have

$$
y(t) \leqslant c B t-\frac{1}{2} m t^{2} \text { for } 0 \leqslant t \leqslant s .
$$

But if we choose $c \in \mathbf{R}^{+}$so that $c<c_{1}:=\left(2 m y_{0}\right)^{1 / 2} / B$, then

$$
c B t-\frac{1}{2} m t^{2}<y_{0} \text { for all } t>0
$$

whence $s=\infty$. By choosing, in addition, $c<c_{2}:=\frac{1}{2} m t_{0} / B$, we ensure, using (A.1) that $y\left(t_{0}\right)<0$. Since $y^{\prime}(0)=c g(0)>0$, this means that the graph of $y$ has a maximum at a point $\tau \in\left(0, t_{0}\right)$. Hence we have $\eta:=y(\tau) \in\left(0, y_{0}\right)$ and $y^{\prime}(\tau)=0$.

\section{REFERENCES}

1. D. G. Aronson, M. G. Crandall and L. A. Peletier, Stabilization of solutions of a degenerate nonlinear diffusion problem, Nonlinear Anal. 6 (1982), 1001-1022.

2. D. R. Atthey, A finite difference scheme for melting problems, J. Inst. Math. Appl. 13 (1974), 353-366.

3. M. Bertsch, P. de Mottoni and L. A. Peletier, Degenerate diffusion and the Stefan problem, Nonlinear Anal. 8 (1984), 1311-1336. 
4. A. Friedman, One dimensional Stefan problems with nonmonotone free boundary, Trans. Amer. Math. Soc. 133 (1968), 89-114.

5. B. H. Gilding, Hölder continuity of solutions of parabolic equations, J. London Math. Soc. 13 (1976), 103-106.

6. A. M. Meirmanov, The structure of generalized solution of Stefan problem. Periodic solution, preprint.

7. O. A. Oleinik, A method of solution of the general Stefan problem, Soviet Math. Dokl. 1 (1960), 1350-1353.

8. M. Ughi, A melting problem with a mushy region: qualitative properties, Preprint $\mathrm{Nr}$. 1982-83/11, Istit. Mat. "U. Dini”, Univ. of Florence.

Mathematical Institute, University of Leiden, Leiden, The Netherlands (Current address of M. Bertsch and L. A. Peletier)

Dipartimento di Matematica, II Universita di Roma - Tor Vergata, Roma, Italy (Current address of $\mathbf{P}$. de Mottoni) 